\title{
PROCESS INDUCED DEFORMATIONS AND RESIDUAL STRESSES IN CURVED COMPOSITE PARTS: A PARAMETRIC ANALYSIS
}

\author{
Enrico Zappino $^{1 *}$, Marco Petrolo ${ }^{1}$, Navid Zobeiry ${ }^{2}$ and Erasmo Carrera ${ }^{1}$ \\ ${ }^{1}$ MUL $^{2}$ Group, Department of Mechanical and Aerospace Engineering, Politecnico di Torino, Corso \\ Duca degli Abruzzi 24, 10129 Torino, Italy \\ ${ }^{2}$ Materials Science \& Engineering Department, University of Washington, Seattle, 98195, USA \\ *enrico.zappino@polito.it
}

The manufacturing process of composite materials leads to residual stresses and process-induced deformations. These defects originate from the thermoset resin's curing process, and the thermoelastic loads originated by the curing cycle [1]. The extent of residual stresses and deformations can be affected by many parameters, e.g., stacking sequence, part geometry, curing cycle, toolpart interaction, tool material. Curved parts, such as L-shaped components, undergo severe residual deformations, referred to as spring-in angle. The prediction of these phenomena requires the use of refined numerical models. The three-dimensional nature of the problem and the multiple physical fields involved make classical models ineffective. The use of solid elements leads to accurate results but requires a very high computational cost.

An efficient numerical approach for predicting the spring-in angle of composite parts has been presented recently [2]. The use of higher-order finite elements has been demonstrated to be as accurate as a three-dimensional model with a fraction of the computational cost.

The present work exploits the computational efficiency of this model [3] to investigate the effects of many parameters on residual stresses and process-induced deformations of L-shaped composite parts. A large simulation matrix has been considered, including a combination of different stacking sequences, tool materials, curing cycles, and part geometries. The effects of each of those parameters on the spring-in angle have been evaluated. The use of a layer-wise model has allowed the effects of each parameter on the residual stresses to be investigated. The large number of simulations has led to an exhaustive understanding of the phenomenon providing a few best practices for reducing defects during the manufacturing of composite parts. The large amount of data produced could be the basis for developing future surrogate models, such as neural networks, that can play a crucial role in virtual manufacturing applications.

\section{References}

[1] Baran, I., Cinar, K., Ersoy, N.,Akkerman, R. and Hattel J.H. (2017) A Review on the Mechanical Modeling of Composite Manufacturing Processes. Archives of Computational Methods in Engineering, 24, 365-395.

[2] Zappino, E., Zobeiry, N., Petrolo, M., Vaziri, R., Carrera, E., Poursartip, A. (2020) Analysis of process-induced deformations and residual stresses in curved composite parts considering transverse shear stress and thickness stretching. Composite Structures, 241, 112057.

[3] Carrera E, Cinefra M, Petrolo M and Zappino E (2014) Finite element analysis of structures through Unified Formulation. John Wiley \& Sons. 